\title{
MORPHOLOGICAL AND MOLECULAR RATES OF EVOLUTION IN POST-PALEOZOIC ECHINOIDS
}

SMITH $^{*}$, Andrew B., Department of Palaeontology, British Museum (Natural Hisory), Cromwell Road, London SW7 5BD, U.K.; CHRISTEN, R., URA 671 CNRS, Station Zoologique, Villefranche-sur-Mer 06230, France.

Phylogenetic analysis of a data matrix of 86 skeletal characteristics, and of the first 400 bases of the $5^{\prime}$ end of the large subunit ribosomal RNA gene produce congruent cladograms for 11 extant echinoids. Based on these cladistic analyses the great majority of morphologic and molecular apomorphies can be placed in one of 18 independent geological time intervals. When the cladogram is calibrated using the fossil record of postPaleozoic echinoids, rates of molecular change in 28S rRNA can be calculated and compared with estimated rates of morphological evolution.

Morphological and molecular apomorphies acquired in each time interval both correlate moderately strongly with time elapsed, but less strongly with one another. As expected, morphological rates vary considerably over time and between sister groups, but so too do molecular rates. When averaged over all echinoids studied, the number of morphological apomorphies accrued yields a slightly higher Spearman Rank correlation coefficient with time elapsed than does the number of molecular apomorphies accrued. This is because there is a three-fold difference in the rate of molecular change amongst the echinoid lineages studied. 